\title{
Surface plasmon resonance-enhanced light interaction in an integrated ormocomp nanowire
}

\author{
Charusluk Viphavakit ${ }^{1} \cdot$ Sakoolkan Boonruang ${ }^{2} \cdot$ \\ Christos Themistos ${ }^{3} \cdot$ Michael Komodromos $^{3}$ • \\ Waleed S. Mohammed ${ }^{4}$ B. M. Azizur Rahman ${ }^{1}$
}

Received: 9 August 2015/Accepted: 3 March 2016/Published online: 20 April 2016

(C) The Author(s) 2016. This article is published with open access at Springerlink.com

\begin{abstract}
An integrated ormocomp nanowire coated with gold metal layer is proposed and its optical characteristics with the effect of surface plasmon resonance (SPR) are studied. The integrated rib-like nanowire has a trapezoidal shape with sidewall angles of $75^{\circ}$. It is coated with $50 \mathrm{~nm}$ gold layer to introduce the SPR and enhance the evanescent field in the sensing region located at the dielectric/metal interface. The possible field modes, the normalized power confinement, and the SPR peak position of the nanowire are studied over the wavelength and the metal thickness by using the full-vectorial $\mathbf{H}$-field FEM in quasi-TM mode. The attenuation coefficient of the nanowire, the SPR peak wavelength, and the wavelength shift is experimentally extracted for three different cladding materials. The redshift of the supermode coupling between the dielectric mode and the anti-symmetric supermode is observed with the higher cladding-index and larger metal thickness. The improvement of the power confinement in the sensing region with the SPR effect is ten times $(10 \times)$ better than a previous similar study.
\end{abstract}

This article is part of the Topical Collection on Optical Wave and Waveguide Theory and Numerical Modelling, OWTNM' 15.

Guest edited by Arti Agrawal, B. M. A. Rahman, Tong Sun, Gregory Wurtz, Anibal Fernandez and James R. Taylor.

Charusluk Viphavakit

charusluk.v@gmail.com

1 School of Mathematics, Computer Science and Engineering, City University London, Northampton Square, London EC1V OHB, UK

2 Photonics Technology Laboratory, Thailand National Electronics and Computer Technology Center (NECTEC), Klong Luang, Pathumthani 10120, Thailand

3 Department of Electrical Engineering, Frederick University, 7 Frederickou Str., 1036 Nicosia, Cyprus

4 Center of Research in Optoelectronics, Communication and Control System (BU-CROCCS), Bangkok University, Paholyotin Rd., Klong Luang, Pathumthani 10120, Thailand 
Keywords Polymer waveguides - Subwavelength structures - Integrated optics devices · Optical design and fabrication

\section{Introduction}

Nanowires are one-dimensional structures exhibiting a high aspect ratio $(\geq 1000)$. They are considered as a good candidate for nanotechnology applications due to their unique properties of high surface-to-volume ratio (Shen et al. 2009; Yun et al. 2003; Themistos et al. 2009). In photonics, nanowires are used as optical waveguides, and particularly silicon-on-insulator (SOI) based nanowires have been used as optical sensors because they have the advantage of high index contrast and low optical power losses (Dell'Olio and Passaro 2007; Sirbuly et al. 2006; MacCraith et al. 1991; Lou et al. 2005; Wang and Dai 2010).

Recently, polymer nanowires are alternatively being used as optical waveguides in sensing applications due to their attractive features. Polymer nanowires have better mechanical flexibility compared to semiconductor nanowires and they are biocompatible materials which can have different functional dopants (Adhikari and Majumdar 2004; Viphavakit et al. 2014b). Their fabrication process can be simpler than those of glass nanowires. Hence, they can be used in a variety of possible sensing applications. By exploiting their chemical selectivity, polymer nanowires can be used to detect a wide range of chemical compounds including toxics gases, metal ions, and DNA (Huang et al. 2003; Wang et al. 2002; Ramanathan et al. 2005). Polymer nanowires have been studied for use as chemical sensors, biosensors, organic light emitting diodes, and organic solar cells (Huang et al. 2003; Liu et al. 2004; Ramanathan et al. 2005). It has been reported that polymer nanowires can be used in humidity sensing and $\mathrm{NO}_{2}$ and $\mathrm{NH}_{3}$ detection with $30 \mathrm{~ms}$ response time and high sensitivity ( $\mathrm{Gu}$ et al. 2008). The use of fluorescent polymer nanofibrous to detect the metal ions $\left(\mathrm{FE}^{3+}\right.$ and $\mathrm{Hg}^{2+}$ ) has been reported (Wang et al. 2002). The polymer nanowires can also be modified as electrical conductors and used to detect $\mathrm{NH}_{3}$ gas at concentrations as low as 0.5 ppm (Liu et al. 2004).

The attenuation loss of nanowires can be due to volume absorption and surface scattering (Tien 1971). The polymer nanowires examined in earlier work (Viphavakit et al. 2014a), with dimensions similar to those of the structures presented in this paper, exhibited surface scattering, caused by the surface roughness which has been considered as the dominant factor to enhance the evanescent fields and the sensitivity. However, the sensitivity of polymer nanowires can be further improved by coating a thin metal layer on the top surface which introduces surface plasmon resonance (SPR) (Fukuda et al. 2010). It has been reported that the optical transmission through the sub-wavelength holes in the metal film can be increased by an additional factor of 10 (Krishnan et al. 2001). The use of colloidal Au as a nanoparticles tag for the detection of DNA hybridization has shown great improvement in the sensitivity compared to those without Au (He et al. 2000). The use of localized surface plasmon resonance (LSPR) to enhance the sensitivity of the waveguides for biosensors has also been addressed (Byun et al. 2005; Kim et al. 2006). A low propagation loss can be achieved by exhibiting the long-range surface plasmon polarition (LRSPP) in thin gold stripes embedded in polymer (Nikolajsen et al. 2003). The sensitivity of the SPR sensor integrated with a Mach-Zehnder interferometer for glycerin-water solutions is significantly improved to $5.5 \times 10^{-8} \mathrm{RIU}$ per $0.01^{\circ}$ phase change (Wu et al. 2004). 
Also with SPR, it has been reported that $2 \mathrm{ng}$ of $\mathrm{nCG}$ presence in $1 \mathrm{ml}$ of $1 \%$ bovine serum albumin solution can be detected with a small refractive index change which is $<1.2 \times 10^{-6}$ (Dostalek et al. 2001).

The design of gold coated polymer nanowires, the characterization of their guided modes using finite element method (FEM) and the experimental measurements of their optical characteristics obtained are presented in the this work. The polymer nanowires examined are considered to be trapezoidal, as shown in Fig. 1a, with their height to be about $0.5 \mu \mathrm{m}$ and the top and bottom widths, 1.0 and $1.5 \mu \mathrm{m}$, respectively, with the dimensions of their guided area, being much smaller than the core diameter of single mode fibers (SMF). Therefore, the polymer nanowires with the integrated optics structure are designed in order to minimize the power losses occurred when launching the light from the source into the nanowires. Also, the integrated optics structure allows the nanowires to be easily used with other optical devices for sensing applications. In this paper, the polymer nanowires coated with a thin metal layer are intended to be used as optical transducers to detect the change of effective index when the cladding-index is varied.

The nanowires were fabricated using ormcomp, which is a hybrid-polymeric material. It is a UV-curable material with refractive index of 1.52. The fabrication of the polymer nanowires used in this study is implemented by the nano-imprint method which has been described earlier (Viphavakit et al. 2014a). The gold metal layer is deposited on the surface of the nanowires using sputtering where its refractive index is wavelength dependent.

In the present work, a full-vectorial H-field FEM is used to study the propagation characteristics of the guided modes, their normalized power confinement in the sensing region, and their resonance peak over the operating wavelength with the thickness of metal layer, and the cladding-index. The sensing area is considered to be the cladding/gold interface.

The experimental part consists of the attenuation coefficient extraction, the determination of the SPR peak wavelength and its shift. The cladding materials used are water and iso-propanol solution with different volume ratios. A comparison of the SPR peaks at some specific cladding-indices between the simulation results and the experimental results based

(a)

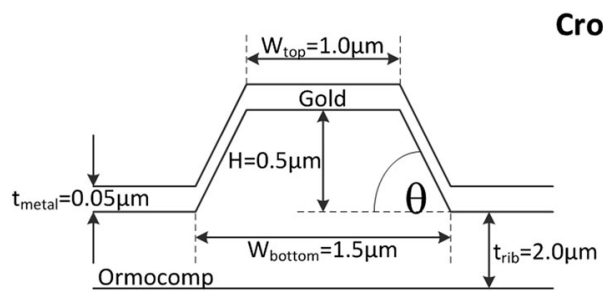

Cross section

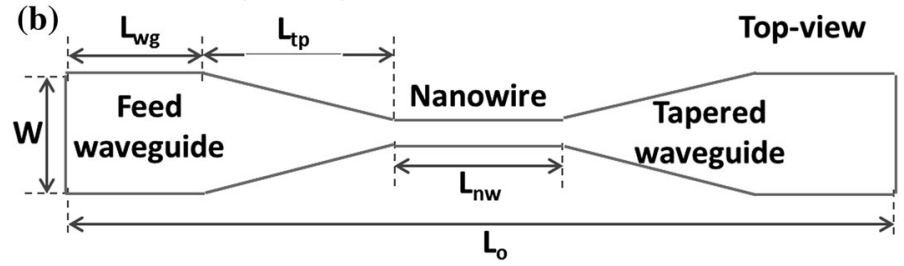

Fig. 1 a A cross-section of the nanowire structure coated with thin metal layer. The rib-shaped nanowire has non-vertical sidewalls. The structure height is $0.5 \mu \mathrm{m}$. The metal layer has the thickness of $50 \mathrm{~nm}$. b A top-view of an integrated nanowire connected to a feed waveguide and a tapered waveguide 
on the metal thickness are also presented. With the SPR, the normalized power confinement in the sensing region is significantly increased at the resonance wavelength. Hence, the sensitivity of the ormocomp nanowires coated with gold is improved compared to those without gold layer.

\section{Design of the nanowires}

The ormocomp polymer nanowires were fabricated using the nano-imprint method (Viphavakit et al. 2014a). A silicon nanowire is used as a master mold in the nano-imprint process. The master mold silicon nanowire results in slanted sidewalls from the anisotropic wet etching along the crystal plane. Consequently, the non-vertical sidewalls ormocomp nanowires are considered and their optical imaging and optical properties including the attenuation coefficient are studied. The surface roughness, as a result of the fabrication process, enhances the sensitivity of the non-vertical sidewalls ormocomp nanowires (Viphavakit et al. 2014a). The effect of the surface roughness is not strong enough to maximize the sensitivity of the nanowires. However, the sensitivity can be improved by introducing surface plasmon resonance at the top surface of the nanowires where the evanescent field exists.

In this paper, we present the effect of surface plasmon resonance (SPR) to the nonvertical sidewalls ormocomp nanowires. A thin gold layer with the thickness of $50 \mathrm{~nm}$ is coated on the nanowires using the sputtering technique. Due to the slanted structure, the gold layer covers both the top surface and the sidewalls. A cross-section of the non-vertical sidewalls nanowire coated with gold layer is presented in Fig. 1a. The ormocomp nanowire structure has height $0.5 \mu \mathrm{m}$. The top and bottom widths of the trapezoidal core are 1.0 and $1.5 \mu \mathrm{m}$, respectively. The ormocomp nanowires have rib-shaped structure due to the leftover ormocomp layer from the stamping process in the nano-imprint (Viphavakit et al. 2014a). The sidewall angle $(\theta)$ is measured to be around $75^{\circ}$. The gold layer at the top

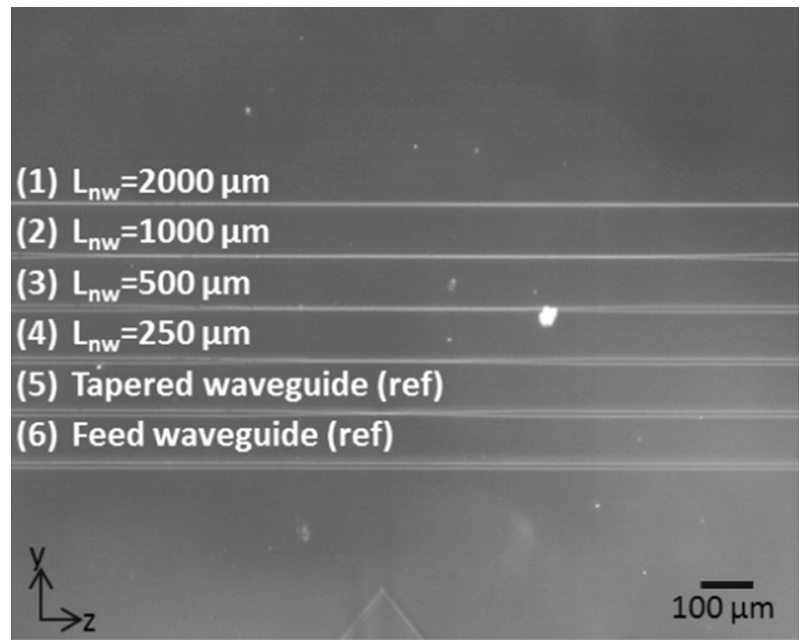

Fig. 2 Optical images of a set of nanowires consisting of the feed waveguide, the tapered waveguide, and four nanowires of different lengths 
surface has a uniform thickness of $50 \mathrm{~nm}$. The nanowires are designed to be easily used with other optical devices, such as optical couplers, by having feed waveguides and tapered waveguides attached to their ends as shown in Fig. $1 \mathrm{~b}$.

The integrated nanowire structure shown in Fig. 1 has length $L_{0}=5000 \mu \mathrm{m}$. It consists of a feed waveguide $\left(L_{w g}\right)$, a tapered waveguide $\left(L_{t p}\right)$ with a length of $270 \mu \mathrm{m}$ and the nanowire $\left(L_{n w}\right)$ with a length in a range of 250-2000 $\mu \mathrm{m}$. To study the optical properties of the nanowire such as the attenuation coefficient $\left(\alpha_{n w}\right)$, a set of nanowires containing nanowires with different lengths is designed and fabricated, as presented in Fig. 2. The nanowire set has one reference feed waveguide, one reference tapered waveguide, and four nanowires with lengths of 250, 500, 1000, $2000 \mu \mathrm{m}$.

Even though the sidewalls of the trapezoidal shape nanowires are coated with a layer of gold, the sidewall angle is large enough to neglect the thickness of the gold layer at the sidewalls. Hence, the surface plasmon resonance effect is dominant at the top surface (TM mode) while the effect of the surface plasmon resonance at the sides is negligible (TE mode). It was also observed experimentally that there was no significant TE mode SPR signal.

\section{Simulation results}

Light propagation along the metal-coated nanowire can be implemented via dielectric and plasmonic modes mainly propagating in the core region and the metal/dielectric interface, respectively. Plasmonic mode is generated when the momentum of photons matches with the momentum of the plasmons. The first order approximation resonance condition at the semi-infinite dielectric/metal interface is given by (Raether 1988);

$$
\beta_{s p}=\frac{2 \pi}{\lambda} \sqrt{\frac{\varepsilon_{m}^{\prime}(\lambda) \varepsilon_{c}}{\varepsilon_{m}^{\prime}(\lambda)+\varepsilon_{c}}}
$$

where $\beta_{s p}$ is the propagation constant for SPR mode. The resonance occurs when the propagation constant of the guided mode equals to $\beta_{s p}$, where $\lambda$ is the operating wavelength in free space. Note that $\varepsilon_{m}^{\prime}$ is the real part of the dielectric constant of the metal, which is a wavelength dependent parameter, and $\varepsilon_{c}$ is the dielectric constant of the cladding material $\left(\varepsilon_{c}=n_{c}^{2}\right.$ ). The surface plasmon resonance exists when $\varepsilon_{m}^{\prime}$ is negative and its magnitude is larger than $\varepsilon_{c}\left(\varepsilon_{m}^{\prime}<0\right.$ and $\left.\left|\varepsilon_{m}^{\prime}\right|>\varepsilon_{c}\right)$. As mentioned above the ormocomp nanowires are coated with a gold layer of thickness of about $50 \mathrm{~nm}$. For the SPR at the finite dielectric/ metal interface, the analytical form of the resonance condition can be calculated from the incident light reflectivity where the metal thickness is the key parameter (Raether 1988). However, for the SPR on the metal-coated nanowire, the finite element method (FEM) is used instead of the analytical approach for a 2-dimensional waveguide and the metal thickness is also taken into consideration when $\beta_{s p}$ is calculated.

The full-vectorial $\mathbf{H}$-field finite element formulation has been used to study and analyze the behavior of the ormocomp nanowires with the effect of the SPR in order to determine the propagation characteristics of the fundamental optical mode. The functional used to solve the problems is related to the standard eigenvalue problems, and the modal characterization is obtained by minimizing the functional as shown in Eq. 2 (Rahman and Davies 1984). 


$$
\omega^{2}=\frac{\int\left[(\nabla \times \boldsymbol{H})^{*} \cdot \varepsilon^{-1}(\nabla \times \boldsymbol{H})+p(\nabla \cdot \boldsymbol{H})^{*} \cdot(\nabla \cdot \boldsymbol{H})\right] d x d y}{\int \boldsymbol{H}^{*} \cdot \mu \boldsymbol{H} d x d y}
$$

where $\mathbf{H}$ is the full vectorial magnetic field, * represents the complex conjugate and transpose, $\omega$ is the angular frequency of the wave, $\omega^{2}$ is the eigenvalue, and $\varepsilon$ and $\mu$ are the permittivity and permeability, respectively. In this work, 125,000 first order triangles are used to represent the one half of the nanowire structure by exploiting the existing one-fold symmetry. The main advantage of the FEM over the other numerical methods is that it can incorporate triangles of different shapes and sizes to achieve numerical efficiency. In our case, vertical resolution of $0.8 \mathrm{~nm}$ or better is achieved for the $50 \mathrm{~nm}$ thick metal layer.

In the numerical simulations the ormocomp nanowires are assumed to be coated with a $50 \mathrm{~nm}$ gold layer only at the top surface. Therefore, only the quasi-TM mode $\left(\mathrm{H}_{\mathrm{x}}\right)$ is considered. For the quasi-TM mode, the dominant electric field component, $\mathrm{E}_{\mathrm{y}}$, is perpendicular to the dielectric/metal interface. The possible mode fields and power confinement of the ormocomp nanowires with the effect of SPR are studied over the wavelength for different cladding materials. Ormocomp is a transparent hybrid-polymer material with the refractive index $\mathrm{n}=1.52$. The complex refractive index of gold $(\tilde{n}=n+i \kappa)$ is dependent on the operating wavelength (Wooten 2013). We consider the visible region $(400-700 \mathrm{~nm})$ in this work. According to the experimental work, water $(\mathrm{n}=1.333)$ and two different volume ratios of iso-propanol solution $(\mathrm{n}=1.351$ and $\mathrm{n}=1.365)$ are considered as the cladding materials. As it was mentioned above, the ormocomp nanowires were fabricated using nano-imprint method and they have trapezoidal shape cross-section, with the fixed dimensions of $0.5 \mu \mathrm{m}$ height, $1.5 \mu \mathrm{m}$ bottom-width, and $1.0 \mu \mathrm{m}$ top-width (Viphavakit et al. 2014a).

The two possible optical modes in the nanowire structures are the dielectric mode inside ormocomp region and the plasmonic mode at the dielectric/metal interface. These two modes can be clearly separated or coupled with each other to create a supermode depending on the parameters of the nanowire structure, especially the height of the nanowire. In the case of nanowires with height $0.5 \mu \mathrm{m}$, the coupled dielectric-plasmonic

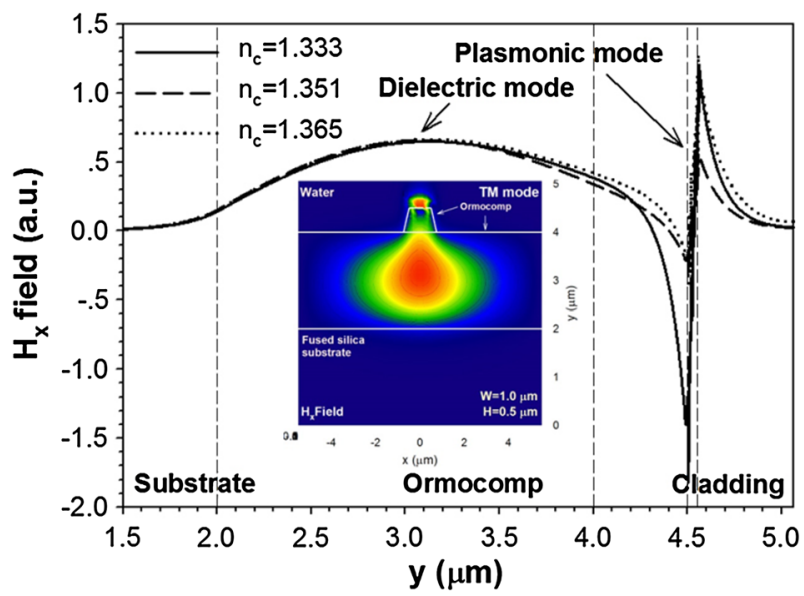

Fig. 3 The $H_{x}$ of the coupled dielectric-plasmonic mode along the $y$-axis for three different cladding indices. The Inset shows the modal field profile showing and the coupling between the dielectric mode in the slab layer and the plasmonic mode in water cladding 
mode is shown in Fig. 3, where the supermode coupling between the dielectric mode and the odd-like plasmonic supermode can be clearly seen from the $\mathrm{H}_{\mathrm{x}}$ profile along the y-direction. The inset shows the $\mathrm{H}_{\mathrm{x}}$-field contour plot of the coupled mode with water cladding.

Variations of the effective indices of the fundamental $\mathrm{H}_{\mathrm{x}}$ modes in three different cladding mediums with respect to the operating wavelength are shown in Fig. 4. The effective indices of the optical mode decrease when the wavelength is increased regardless of the cladding-index because the wavelength becomes larger compared to the dimension of the nanowire. However, from the characteristics of the effective indices, particularly the peak at some specific wavelengths, it can be used to identify the SPR wavelengths. Effective index of the plasmonic mode is normally higher than that of the fundamental dielectric mode. Hence, the effective index of the coupled dielectric-plasmonic mode occurring at a certain wavelength is slightly higher than that of the dielectric mode alone.

At the wavelength where the SPR occurs, the power confinement in the sensing region which is at the gold/cladding interface also increases because the evanescent field at the interface is enhanced. The variation of the normalized power confinement in the sensing area with the wavelength is shown in Fig. 5. The power confinement in the sensing region of the gold-coated ormocomp nanowire in water-cladding for quasi-TM mode is 10 times higher than the ormocomp nanowire with the same parameters but without gold layer which was examined in our previous study (Viphavakit et al. 2014a).

The resonance peak wavelength at which the SPR signal occurs for each cladding material can be recognized from the peak in both the effective index and power confinement graphs. In case of water-cladding, the SPR signal is obtained at $\lambda=551 \mathrm{~nm}$. However, the plasmonic modes in iso-propanol solution are observed at $\lambda=576$ and $\lambda=590 \mathrm{~nm}$ for the cladding-indices of 1.351 and 1.365 , respectively. As the claddingindex increases, a redshift of the SPR peak wavelength is observed. Besides the cladding material, the thickness of the metal layer is another important parameter which also affects the resonance peak position.

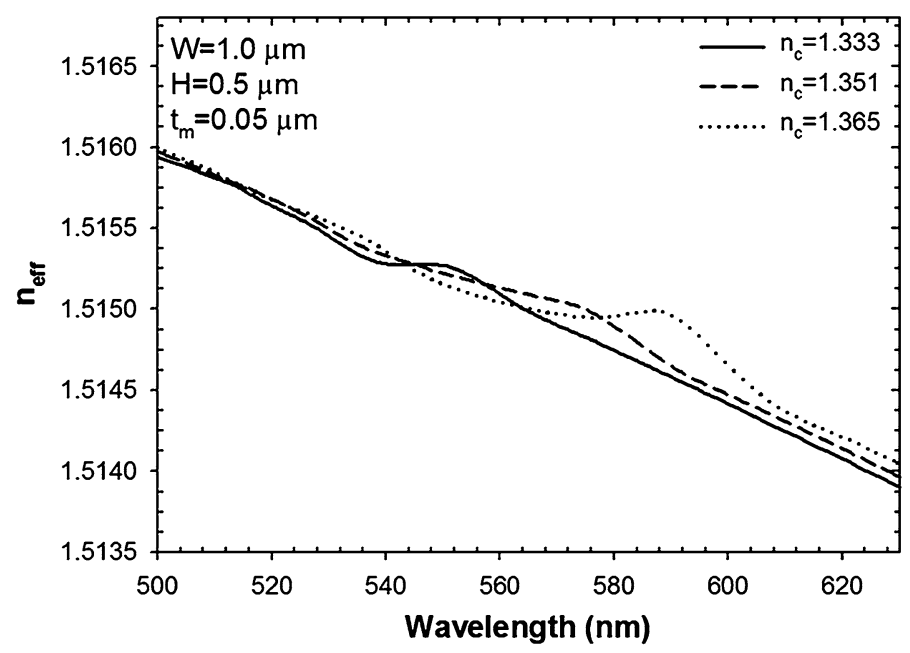

Fig. 4 Variation of effective index of the gold-coated ormocomp nanowire for different cladding materials with respect to the operating wavelength. The peaks indicate the wavelengths where the SPRs are introduced 


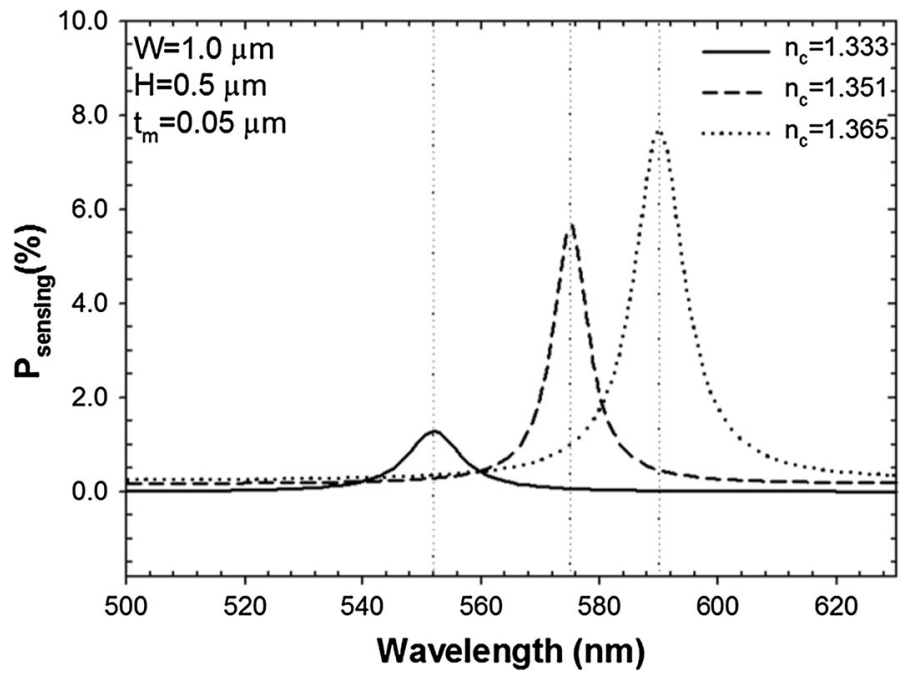

Fig. 5 Variation of normalized power confinement in the sensing region of a nanowire for different cladding-indices with the operating wavelength. The highest power confinement is obtained at the SPR wavelength

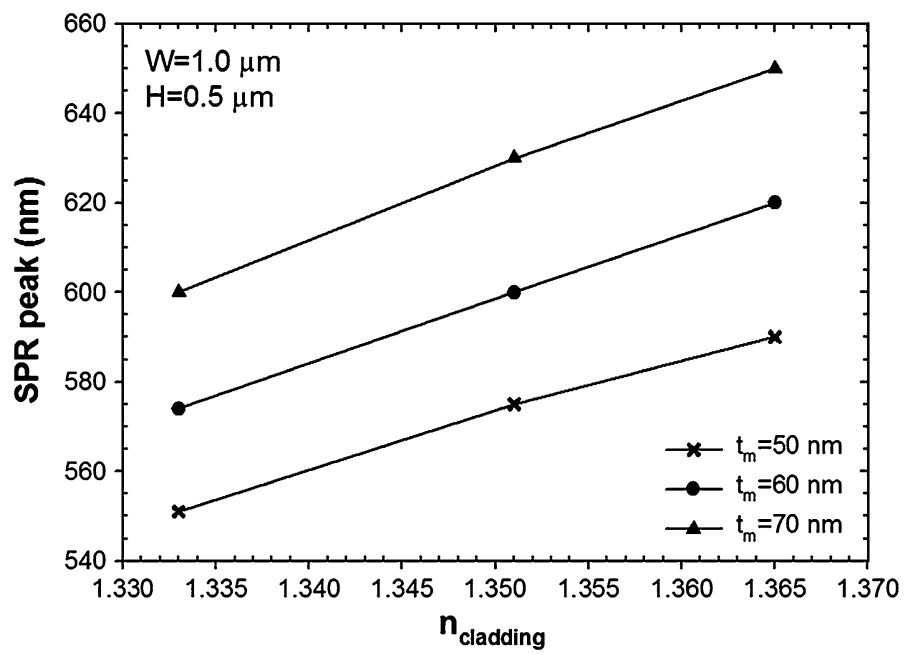

Fig. 6 Variation of SPR peak wavelength of the ormocomp nanowire in three different cladding materials with the gold thickness of 50,60, and $70 \mathrm{~nm}$. The redshift occurs for the antisymmetric modes when the thickness of gold layer is increased

For gold-coated nanowires, the SPR occurs at both the nanowire/metal and cladding/ metal interfaces. These two plasmonic modes can be coupled to the supermode of symmetric or antisymmetric resonance. With thicker metal layer, the antisymmetric surface plasmon has a redshift while the symmetric surface plasmon obtains a blueshift (Tang and He 2011). In this paper, the antisymmetric mode (odd mode) as shown in Fig. 3 is 
considered. The relation between the SPR peak wavelengths for each cladding material with different metal thickness for an odd-plasmonic supermode is shown in Fig. 6.

The metal thicknesses of 50,60, and $70 \mathrm{~nm}$ are considered to check for the resonance peaks and their shifts. At a very thin metal layer, the enhancement of the evanescent fields by SPR is less compared to that with thicker metal layer. However, if the thickness of the metal layer is too large, the coupling between the two surface plasmon modes is weaker. Hence, the power confinement and sensitivity at the cladding/metal interface is decreased. As it is seen in Fig. 6, the redshift of about $25-30 \mathrm{~nm}$ is observed in each cladding-index when the metal thickness is increased by $10 \mathrm{~nm}$.

\section{Experimental results}

The experimental results presented below include the extraction of the attenuation coefficient of the ormocomp nanowires $\left(\alpha_{n w}\right)$ and the analysis of the SPR peak and its shift with the use of different cladding materials. The actual nanowires used in the experiment were fabricated using the nano-imprint method (Viphavakit et al.). The gold layer is coated on the ormocomp nanowires using the sputtering technique. The cladding materials are water $(\mathrm{n}=1.333)$ and two iso-propanol solutions. The iso-propanol solution is a mixture of water and iso-propanol. For this paper, iso-propanol solutions with two different volume ratios, 1:1 and 1:3, are used, yielding refractive indices of 1.365 and 1.351, respectively. The light source used in this experiment is a broadband light source where the operating wavelength is in visible wavelength $(\lambda=400-700 \mathrm{~nm})$.

\subsection{Extraction of attenuation coefficient}

The ormocomp nanowire fabricated by using nano-imprint method has non-vertical sidewalls with the sidewall angle of $75^{\circ}$. Therefore, the gold layer deposited using the sputtering technique at the sidewalls is thinner compared to the top surface due to the sidewall angle. This assumption is confirmed from the experimental work as there is no effect of SPR at the sidewalls (TE mode). The thin metal layer at the top surface introduces the coupling of SPR between the ormocomp/gold and cladding/gold interfaces in TM mode.

The optical setup shown in Fig. 7 is used to obtain the attenuation coefficient of a goldcoated ormocomp nanowire. The spectrometer is used to measure the intensity of the transmittance signals. The white LED connected with a single mode optical fiber is used as a broadband light source $(\lambda=400-700 \mathrm{~nm})$. The input light from the light source is considered to be unpolarized light, so a polarizer is needed in the optical setup in order to be able to select the TM mode signal for the SPR at the top surface of the nanowire. An objective lens with magnification of $20 \times$ is used to focus the light, and also enhance the input signal. In an alignment process, the objective lens with magnification of $10 \times$ is connected with the CCD camera, working as an optical microscope, is used to align the optical fiber and the nanowire on the glass substrate. After a successful alignment, the focused light is coupled into the specific nanowire, and then propagates along the wire. At the end of the nanowire, the output signal is divided into two equivalent beams by the beam splitter. One signal is imaged by the CCD camera. Another output signal is detected by the spectrometer to measure the intensity of the light affected by SPR. The transmittance $(T)$ then can be obtained using the Beer-Lambert law given by 


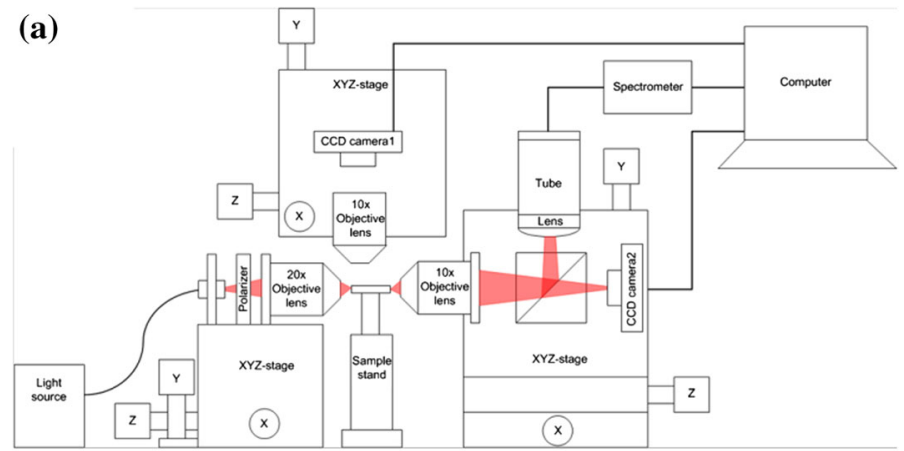

(b)

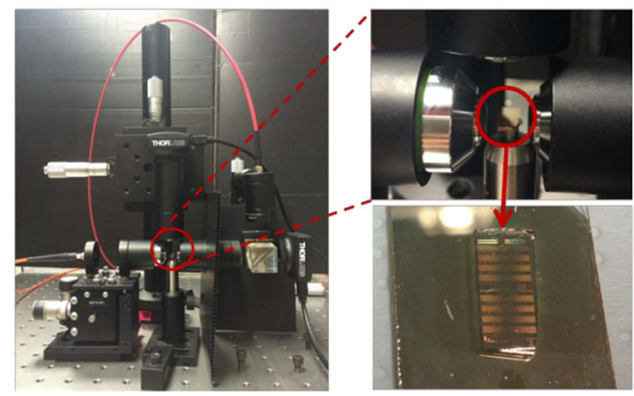

Fig. 7 a Schematic showing the optical setup to measure the transmittance signal. b The actual optical setup and the gold-coated ormocomp nanowires

$$
T=\frac{I}{I_{0}}=e^{-\alpha(\lambda) L}
$$

where $I_{0}$ is the intensity of the incident light, $I$ is the intensity of the transmitted light. $\alpha$ is the attenuation coefficient as a function of wavelength $(\lambda)$ and $L$ is the path length. Air is considered to be the cladding material for the incident light because the SPR absorption wavelength in the air/metal interface is in the IR region the above nanowires are operated in the invisible region.

As mentioned earlier, the nanowire is designed to be integrated with other optical devices, the feed and tapered waveguides at its ends. Therefore, the attenuation coefficient of the integrated nanowire $(\alpha)$, consists of the attenuation coefficients from the feed waveguide $\left(\alpha_{w g}\right)$, the tapered waveguide $\left(\alpha_{t p}\right)$ and the nanowire itself $\left(\alpha_{n w}\right)$. Therefore, the set of integrated nanowires shown in Fig. 2 is needed. The set includes the reference feed waveguide, the reference tapered waveguide, and nanowires with different lengths, so the attenuation coefficient of the nanowire can be extracted individually. The attenuation coefficient of a nanowire $\left(\alpha_{n w}\right)$ can be derived from Eq. 3 as stated below

$$
\alpha_{n w}(\lambda)=\frac{-\alpha_{w g}(\lambda)\left(L_{0}-2 L_{t p}-L_{n w}\right)-2 \alpha_{t p}(\lambda) L_{t p}-\ln \left(T_{n w}\right)}{L_{n w}}
$$

where $L_{0}$ is $5000 \mu \mathrm{m}$ and $L_{t p}$ is $270 \mu \mathrm{m}$. The incident light $\left(I_{0}\right)$ and the transmitted light (I) are the light propagating in the nanowires with air cladding and water cladding, respectively. The transmittance of the integrated nanowires $\left(T_{n w}\right)$ with different lengths, $L_{n w}=250,500,1000$, and $2000 \mu \mathrm{m}$, obtained with the spectrometer is presented in Fig. 8 . 


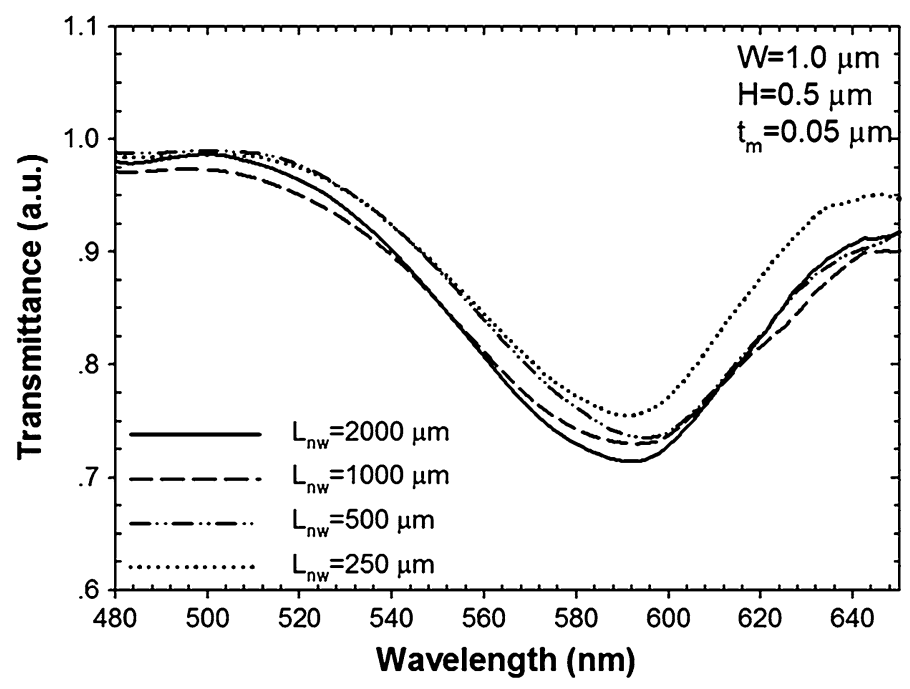

Fig. 8 The transmittance of the gold-coated nanowires with lengths 250, 500, 1000, $2000 \mu$ mer the wavelength in the visible region. The transmittance decreases for longer nanowire due to the SPR absorption

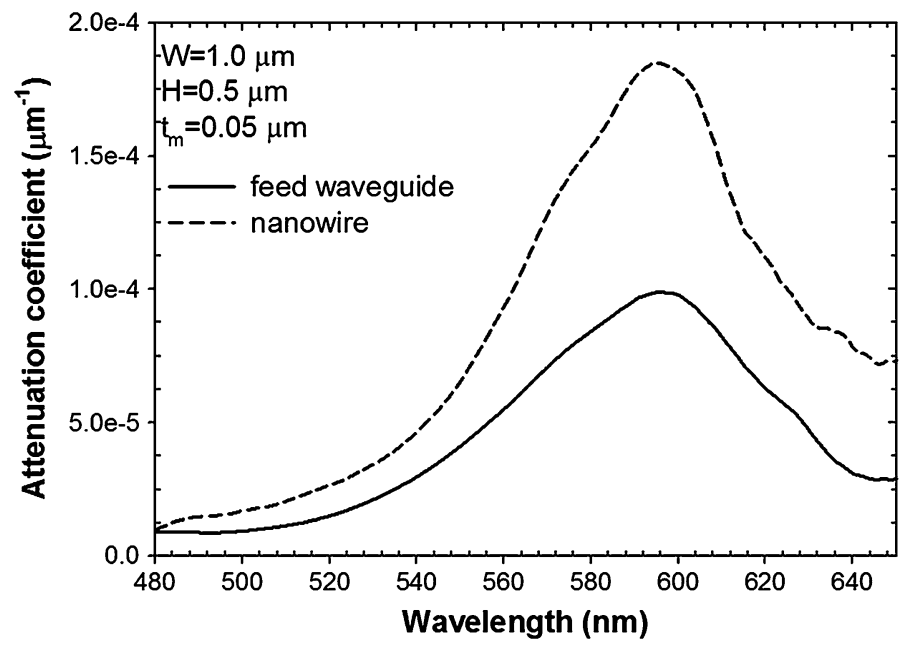

Fig. 9 Variation of the average attenuation coefficient of the feed waveguide and ormocomp nanowires with respect to wavelength for the water cladding. The nanowire has higher SPR absorption compared to the wider feed waveguide

It can be seen that the longest nanowire $\left(L_{n w}=2000 \mu \mathrm{m}\right)$ represented in solid line has the smallest transmittance. The dotted line represents the shortest nanowire $\left(L_{n w}=250 \mu \mathrm{m}\right)$. It has the highest transmittance. It can be concluded that the longest nanowire has the highest SPR absorption. Using the transmittance value from Fig. 8 together with Eq. 4, the attenuation coefficient of the feed waveguide and nanowire can be obtained seperately as presented in Fig. 9. 
The average attenuation coefficient of the nanowires (dashed line) and the feed waveguides (solid line) is presented in Fig. 9. The absorption peak for both the feed waveguide and the nanowires are found to occur at $\lambda=590 \mathrm{~nm}$. The feed waveguide has absorption coefficient of $\alpha_{w g}=1.03 \times 10^{-4} \mu^{-1}$, which is lower than the absorption coefficient of the nanowires $\alpha_{n w}=1.93 \times 10^{-4} \mu \mathrm{m}^{-1}$, since the feed waveguides are multimode and the nanowires are single mode waveguides. In the multimode waveguide the power is distributed in many modes, causing a reduction of optical power in each mode. In addition, each mode has different SPR momentum. Therefore, the absorption peak of the SPR in each mode is located at different wavelength, causing the broadening of the absorption peak in the feed waveguide.

\subsection{Analysis of the SPR peak}

As described earlier in the previous section, the optical modes examined in the nanowires are the coupled supermode between the dielectric mode and the plasmonic mode. In addition, the plasmonic mode itself is also a supermode due to coupling between two surface plasmon modes at the ormocomp/gold and cladding/gold interfaces. It is sensitive to the change of the cladding index which can be studied by investigating the wavelength shift with different refractive indices of cladding materials. The cladding materials used in this study are water $(\mathrm{n}=1.333)$, and the iso-propanol solutions. The solutions are prepared with different volume ratio of iso-propanol to water. With 1:1 volume ratio, the solution has refractive index of 1.365 and the index of 1.351 is measured for the ratio of 1:3. The transmittance is measured again for different cladding materials. Then, the attenuation coefficient of a nanowire showing the SPR peak wavelength for three cladding materials is illustrated in Fig. 10.

As the cladding-index increases, the SPR peak is shifted to a higher wavelength. According to the simulation results, the redshift occurs with the higher refractive index of cladding material.

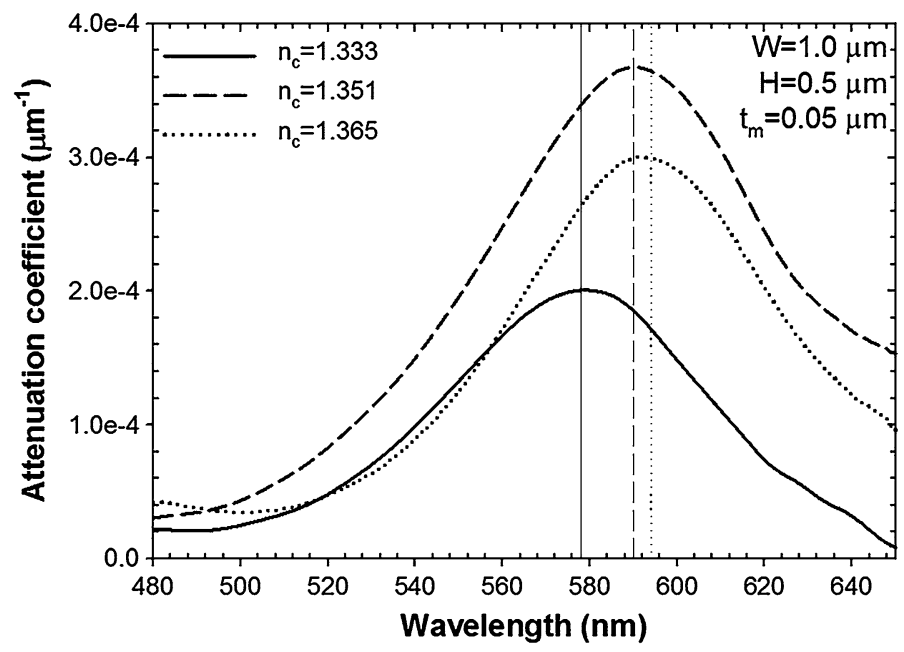

Fig. 10 The graph between the attenuation coefficient of the nanowire for different cladding materials and the wavelength. It shows the SPR absorption peaks and their position. The redshift is observed as the cladding-index increases 
The attenuation coefficient of the gold-coated nanowires $\left(\alpha_{n w}\right)$ is affected by three factors which are the scattering $\left(\alpha_{\text {scattering }}\right)$ from the surface roughness, the SPR $\left(\alpha_{S P R}\right)$ from the thin gold layer and the coupling loss $\left(\alpha_{\text {coupling }}\right)$ from light source coupling into the nanowire. The attenuation coefficient of the gold-coated can be derived as presented below.

$$
\alpha_{n w}=\alpha_{\text {scattering }}+\alpha_{S P R}+\alpha_{\text {material absorption }}
$$

The attenuation coefficient of the gold-coated nanowires increases when increasing the refractive index of the cladding materials, unlike the attenuation coefficient of the noncoated ormocomp nanowires which is reduced when the cladding index increases (Viphavakit et al. 2014a). Therefore, the SPR is the dominant factor that affects the attenuation coefficient in the gold-coated ormocomp nanowires while the scattering from the surface roughness is the main factor for the non-coated nanowires (Viphavakit et al. 2014a). The power loss from material absorption can be negligible because ormocomp has very low absorption in the visible region (Gruetzner et al. 2014).

The average SPR peak wavelength for those three cladding materials is shown in Fig. 11. The comparison of the peak position between the simulation and experimental results is also presented in Fig. 11. From the experimental work, the SPR peak wavelength for the water cladding $(\mathrm{n}=1.333)$ was found to be about $\lambda=578 \pm 0.5 \mathrm{~nm}$. For isopropanol solution cladding with refractive index of 1.351 the peak position is at $\lambda=590 \pm 0.9 \mathrm{~nm}$. For the largest cladding-index in this work $(\mathrm{n}=1.365)$, the SPR is observed at the wavelength of $\lambda=595 \pm 1.7 \mathrm{~nm}$. The SPR peak from the experiment is compared with the $55 \mathrm{~nm}$ gold-coated nanowire in the simulation. The comparison shows the same trend of the peak redshift with the cladding index. However, the effect from the large surface roughness due to the fabrication process has to be taken into account for the

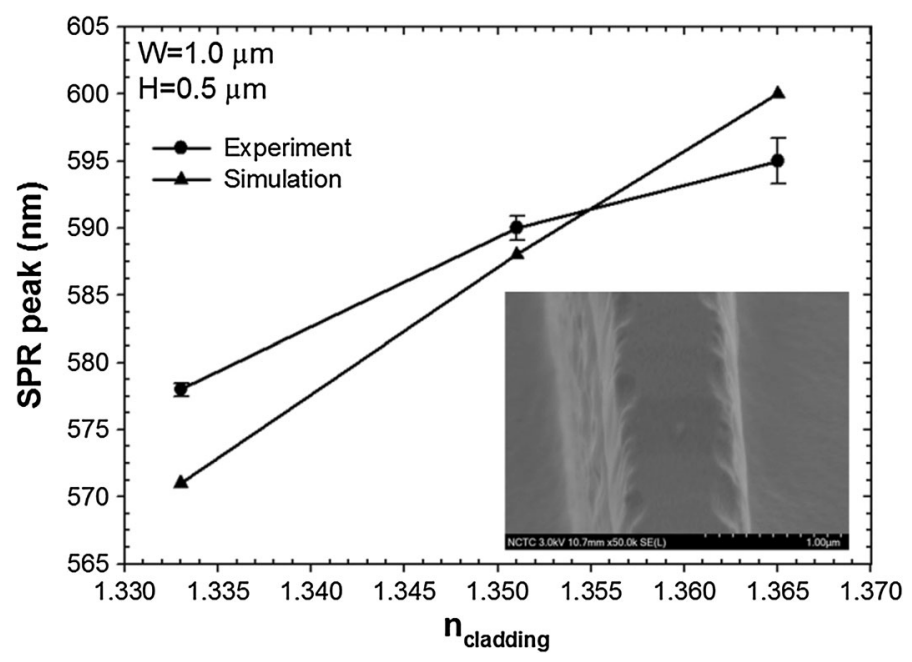

Fig. 11 The comparison of the SPR peak wavelength between the experimental result and the simulation result at three cladding materials. The peak position of the nanowire coated with metal thickness of $55 \mathrm{~nm}$ is the best match with the experimental work. An inset shows the surface roughness of the gold coated nanowire 
experimental works. The surface roughness of the nanowire is about $0.1-0.2 \mu \mathrm{m}$ as shown in the inset of Fig. 11.

\section{Conclusion}

The effect of SPR on the integrated ormocomp nanowire coated with a $50 \mathrm{~nm}$ thick layer of gold is studied both through simulations and experiments. The nanowire is designed to be attached with a feed waveguide and a tapered waveguide at its ends so it can be used as an integrated optics device. The fabricated nanowire has a trapezoidal shape with height $0.5 \mu \mathrm{m}$, bottom width $1.5 \mu \mathrm{m}$ and top width $1.0 \mu \mathrm{m}$. The sidewall angle is about $75^{\circ}$. The cladding materials in this work are water and two different volume ratios of iso-propanol solutions. The operating wavelength is in the visible region $(400-700 \mathrm{~nm})$. The fullvectorial $\mathbf{H}$-field FEM is used in the simulations to study the modal field and the normalized power confinement of the nanowires with vertical resolution of $0.8 \mathrm{~nm}$ in the sensing region. The optical modes presented are the coupled supermode between the dielectric mode in the ormocomp nanowire and the plasmonic supermode. For the thin metal layer coated nanowires, the two plasmonic modes at the ormocomp/gold and cladding/gold interfaces are coupled and form a symmetric or an antisymmetric mode (odd or even mode). In this paper, the odd-like plasmonic supermode is considered. Even though the ormocomp nanowire has a rib-like structure and the optical field is extended inside the slab layer, the plasmonic mode is more significant compared to the dielectric mode at the SPR peak wavelength. The SPR peak wavelength is clearly observed and the redshift occurs when the refractive index of the cladding material is increased for both simulation and experimental results. The peak position also depends on the thickness of the metal layer. With a thicker metal layer, the odd-mode has a red shift. The extracted attenuation coefficient of the nanowire obtained from measuring the transmittance with the spectrometer was found to be $1.93 \times 10^{-4} \mu^{-1}$. It can be seen that the nanowire has higher attenuation coefficient than the feed waveguide which means the nanowire is more sensitive compared to the wide feed waveguide. Also, a longer nanowire has larger SPR absorption.

With the gold layer on the top of a nanowire, SPR is introduced at the interface where the evanescent fields exist and the power confinement is increased by a factor of ten, allowing a larger amount of light interaction with the sensing materials. Hence, the sensitivity of the nanowire is improved. In addition, the SPR is sensitive to the change of the cladding index which is considered to be an important factor in sensing applications.

Open Access This article is distributed under the terms of the Creative Commons Attribution 4.0 International License (http://creativecommons.org/licenses/by/4.0/), which permits unrestricted use, distribution, and reproduction in any medium, provided you give appropriate credit to the original author(s) and the source, provide a link to the Creative Commons license, and indicate if changes were made.

\section{References}

Adhikari, B., Majumdar, S.: Polymers in sensor applications. Prog. Polym. Sci. 29(7), 699-766 (2004)

Byun, K., Kim, S., Kim, D.: Design study of highly sensitive nanowire-enhanced surface plasmon resonance biosensors using rigorous coupled wave analysis. Opt. Express 13(10), 3737-3742 (2005)

Dell'Olio, F., Passaro, V.M.: Optical sensing by optimized silicon slot waveguides. Opt. Express 15(8), 4977-4993 (2007) 
Dostalek, J., Čtyroký, J., Homola, J., Brynda, E., Skalský, M., Nekvindova, P., Špirková, J., Škvor, J., Schröfel, J.: Surface plasmon resonance biosensor based on integrated optical waveguide. Sens. Actuators B Chem. 76(1), 8-12 (2001)

Fukuda, M., Aihara, T., Yamaguchi, K., Ling, Y.Y., Miyaji, K., Tohyama, M.: Light detection enhanced by surface plasmon resonance in metal film. Appl. Phys. Lett. 96(15), 153107 (2010)

Gruetzner, G., Klein, J., Vogler, M., Schleunitz, A.: UV-curable hybrid polymers for optical applications: technical challenges, industrial solutions, and future developments. SPIE MOEMS-MEMS. International Society for Optics and Photonics (2014)

Gu, F., Zhang, L., Yin, X., Tong, L.: Polymer single-nanowire optical sensors. Nano Lett. 8(9), 2757-2761 (2008)

He, L., Musick, M.D., Nicewarner, S.R., Salinas, F.G., Benkovic, S.J., Natan, M.J., Keating, C.D.: Colloidal Au-enhanced surface plasmon resonance for ultrasensitive detection of DNA hybridization. J. Am. Chem. Soc. 122(38), 9071-9077 (2000)

Huang, J., Virji, S., Weiller, B.H., Kaner, R.B.: Polyaniline nanofibers: facile synthesis and chemical sensors. J. Am. Chem. Soc. 125(2), 314-315 (2003)

Kim, K., Yoon, S.J., Kim, D.: Nanowire-based enhancement of localized surface plasmon resonance for highly sensitive detection: a theoretical study. Opt. Express 14(25), 12419-12431 (2006)

Krishnan, A., Thio, T., Kim, T., Lezec, H., Ebbesen, T., Wolff, P., Pendry, J., Martin-Moreno, L., GarciaVidal, F.: Evanescently coupled resonance in surface plasmon enhanced transmission. Opt. Commun. 200(1), 1-7 (2001)

Liu, H., Kameoka, J., Czaplewski, D.A., Craighead, H.G.: Polymeric nanowire chemical sensor. Nano Lett. 4(4), 671-675 (2004). doi:10.1021/n1049826f

Lou, J., Tong, L., Ye, Z.: Modeling of silica nanowires for optical sensing. Opt. Express 13(6), 2135-2140 (2005)

MacCraith, B., Ruddy, V., Potter, C., O’Kelly, B., McGilp, J.: Optical waveguide sensor using evanescent wave excitation of fluorescent dye in sol-gel glass. Electron. Lett. 27(14), 1247-1248 (1991)

Nikolajsen, T., Leosson, K., Salakhutdinov, I., Bozhevolnyi, S.I.: Polymer-based surface-plasmon-polariton stripe waveguides at telecommunication wavelengths. Appl. Phys. Lett. 82(5), 668-670 (2003)

Raether, H.: Surface Plasmons on Smooth Surfaces. Springer, New York (1988)

Rahman, B.M.A., Davies, J.B.: Finite-element solution of integrated optical waveguides. J. Light. Technol. 2(5), 682-688 (1984)

Ramanathan, K., Bangar, M.A., Yun, M., Chen, W., Myung, N.V., Mulchandani, A.: Bioaffinity sensing using biologically functionalized conducting-polymer nanowire. J. Am. Chem. Soc. 127(2), 496-497 (2005)

Shen, G., Chen, P.-C., Ryu, K., Zhou, C.: Devices and chemical sensing applications of metal oxide nanowires. J. Mater. Chem. 19(7), 828-839 (2009)

Sirbuly, D.J., Tao, A., Law, M., Fan, R., Yang, P.: Multifunctional nanowire evanescent wave optical sensors. Adv. Mater. 19(1), 61-66 (2006)

Tang, J., He, S.: Optical Magnetic Response in a Single Metal Nanobrick. arXiv preprint arXiv:1102.2692 (2011)

Themistos, C., Rajarajan, M., Rahman, B.M.A., Grattan, K.T.V.: Characterization of silica nanowires for optical sensing. J. Light. Technol. 27(24), 5537-5542 (2009)

Tien, P.: Light waves in thin films and integrated optics. Appl. Opt. 10(11), 2395-2413 (1971)

Viphavakit, C., Atthi, N., Boonruang, S., Themistos, C., Komodromos, M., Mohammed, W.S., Rahman, B.A.: Realization of a polymer nanowire optical transducer by using the nanoimprint technique. Appl. Opt. 53(31), 7487-7497 (2014a)

Viphavakit, C., Atthi, N., Boonruang, S., Themistos, C., Mohammed, W.S., Kalli, K., Rahman, B.M.A., Komodromos, M.: Characterization of polymer nanowires fabricated using the nanoimprint method. In: SPIE Photonics Europe, pp. 912632-912632-912610. International Society for Optics and Photonics (2014b)

Wang, J., Dai, D.: Highly sensitive Si nanowire-based optical sensor using a Mach-Zehnder interferometer coupled microring. Opt. Lett. 35(24), 4229-4231 (2010)

Wang, X., Drew, C., Lee, S.-H., Senecal, K.J., Kumar, J., Samuelson, L.A.: Electrospun nanofibrous membranes for highly sensitive optical sensors. Nano Lett. 2(11), 1273-1275 (2002)

Wooten, F.: Optical properties of solids. Academic press, Cambridge (2013)

Wu, S., Ho, H., Law, W., Lin, C., Kong, S.: Highly sensitive differential phase-sensitive surface plasmon resonance biosensor based on the Mach-Zehnder configuration. Opt. Lett. 29(20), 2378-2380 (2004)

Yun, M., Myung, N.V., Vasquez, R.P., Wang, J., Monbouquette, H.: Nanowire growth for sensor arrays. In: Optical Science and Technology, SPIE's 48th Annual Meeting 2003, pp. 37-45. International Society for Optics and Photonics 